\title{
A Study on the Prevalence and the Risk Factors of Hepatitis B Virus Infection in Kurdistan Region, Iraq: A Multicenter Study
}

\author{
Nawfal R Hussein', Dildar H Musa², Dawan Jamal Hawezy³, Ferhad MR Ahmed', Fatma Kamal Khalid', \\ Ibrahim A Naqid ${ }^{1 *} \mathbb{D}$, Mahde Saleh Assafi ${ }^{4}$
}

\author{
'Department of Biomedical Sciences, College of Medicine, University of Zakho, Kurdistan Region, Iraq. \\ 2Department of Surgery, College of Medicine, University of Duhok, Kurdistan Region, Iraq. \\ ${ }^{3}$ Department of Surgery, College of Medicine, University of Koya, Kurdistan Region, Iraq. \\ ${ }^{4}$ Department of Biology, College of Science, University of Duhok, Kurdistan Region, Iraq. \\ *Correspondence to: Ibrahim A. Naqid (E-mail: ibrahim.naqid@uoz.edu.krd) \\ (Submitted:07 August 2021 - Revised version received: 26 August 2021 - Accepted:04 September 2021 - Published online: 26 October 2021)
}

\begin{abstract}
Objectives: The aims of this study were to determine the prevalence of HBV infection and investigate the HBV-related risk factors in the Kurdistan region of Iraq.

Methods: Sera samples were collected from 3423 patients visiting three centers and tested for HBV positivity by enzyme-linked immunosorbent assay. A questionnaire was prepared and used by the volunteers who collected data, including age, marital status, and different HBV-related risk factors, through face-to-face interviews.

Results: The mean age of the recruited participants was $28.94 \pm 11.17$ years. In addition, 873/3423 (25.47\%) were males, and 3024/3423 (88.34\%) were married. Furthermore, HBV infection had a prevalence of $1.37 \%$ (47/3243). Multivariate analysis was conducted to identify the predictive factors of HBV infection, which revealed that a history of tattoos and age were predictive factors $(P<0.05)$. We then stratified our data according to sex. No association was found between the various factors and HBV positivity in males. On the other hand, in females, a significant association was found between the history of tattoos or age and HBV positivity $(P<0.05)$.

Conclusion: In conclusion, the prevalence of HBV infection was low. Our study showed that a history of tattooing and older age were predictive of HBV infection. Our results could be used as a basis for local elimination programs.

Keywords: Hepatitis B virus, prevalence, risk factors, ELISA, Iraq
\end{abstract}

\section{Introduction}

HBV infection is a global public health issue that is associated with deleterious consequences, including liver cirrhosis, hepatocellular carcinoma, and hepatic failure ${ }^{1}$. Approximately 500 million people are infected with $\mathrm{HBV}$, with an estimated 750000 deaths annually ${ }^{1}$. The prevalence of HBV infection varies, ranging from $0.5 \%$ in some developed countries to up to $8 \%$ in some East Asian countries ${ }^{1}$. A previous study in Turkey, a neighboring country of Iraq, showed that the prevalence of HBsAg positivity varied markedly from $1 \%$ to $14.3 \%$, according to the geographical region of the study and the recruited samples ${ }^{2}$. While the prevalence of HBV infection was below $1 \%$ in some regions in Iran, studies from Saudi Arabia showed that approximately 3\% of the study populations were infected with the virus ${ }^{3,4}$. The World Health Organization declared an ambitious plan to eliminate viral hepatitis by 2030 . However, it has been previously estimated that only $10 \%$ of $\mathrm{HBV}$-infected patients are aware of their infection ${ }^{1}$. As such, it is essential to study the risk factors associated with viral transmission to come up with a public health plan for combatting HBV infection. Risk factors for HBV include blood and blood product transfusions, pregnancy, being a healthcare worker, tattoos, drug abuse, and high-risk sexual behaviors $^{1,5,6}$. To establish new strategies for the elimination of the virus in Iraq, epidemiological data, including risk factors, should be identified.

Since the distribution of HBV risk factors is important for any public health prevention plan, this study investigated the prevalence of HBV and its associated risk factors in multiple centers in the Kurdistan region, Northern Iraq.

\section{Materials and Methods}

\section{Blood Samples}

Blood samples were collected from volunteers attending Zakho General Hospital, Azadi Teaching Hospital, and Shahid Dr Khalid Teaching Hospital at Koya between January 2019 and February 2021. A 5-cc syringe and needle were used to obtain $5 \mathrm{~mL}$ of blood from the volunteers. To separate the sera, blood samples were centrifuged at $1500 \mathrm{rpm}$ for $3 \mathrm{~min}$. Samples were tested immediately for HBsAg or kept frozen at $-20^{\circ} \mathrm{C}$ until the tests were performed.

\section{Questionnaire}

A questionnaire was prepared and used by each volunteer. Data were collected through face-to-face interviews, including age, marital status, history of blood transfusion, history of dental procedures, history of surgical operation, history of tattoos, and history of regular injections. Regular use of injections was defined as the regular use of over-the-counter injections or any other injections.

\section{Enzyme-Linked Immunosorbent Assay (ELISA)}

$\mathrm{HBsAg}, \mathrm{HBc}-\mathrm{IgM}$, and $\mathrm{HBc}-\mathrm{IgG}$ were analyzed using a commercial ELISA kit (DIA.PRO Diagnostic Bioprobes; Milan Italy), following the manufacturer's instructions. Monoclonal antibodies were fixed to the surface of the micro-wells, and sera samples were added and incubated. Afterward, a secondary monoclonal antibody conjugated with horseradish peroxidase (HRP) was added. Unbound serum proteins and HRP conjugates were washed off. After blocking the 
enzymatic reaction, the substrate was added, and the optical density was measured using an ELISA reader.

\section{Ethics}

The study was approved by the appropriate ethics committee of the College of Medicine, University of Zakho. Written informed consent was obtained from all patients.

\section{Statistics}

Regression analysis was used to assess the risk factors associated with HBsAg. Variables that achieved a $P$-value of less than 0.2 were included in the multivariate study. Backward elimination was used to determine the HBV-related risk factors. All computations were performed using the Minitab 17 (Minitab; Pennsylvania, United States).

\section{Results}

\section{Volunteers}

Sera samples were collected from 3423 patients and tested for HBV positivity. The mean age of the recruited participants was $28.94 \pm 11.17$ years. In addition, 873/3423 (25.47\%) were males, and 3024/3423 (88.34\%) were married (Table 1).

\section{HBV Prevalence and Risk Factors}

The prevalence of HBV infection in the recruited sample was $1.37 \%$ (47/3243). All samples were HBc-IgG-positive, indicating chronic infection. Univariate analysis showed that 14/47 (29.8\%) of the HBV-positive patients had a history of blood transfusion, which was significantly higher compared to the HBV-negative patients $(602 / 3376$ [17.8\%], $P=0.048)$. In addition, HBsAg positivity was significant associated with a history of tattooing $(P=0.039)$ and age $(P=0.003)$ (Table 2). Multivariate analysis was then conducted to identify the predictive factors of HBV infection, which revealed that a history of tattoos and age were predictive factors (Table 2).

We then stratified our data according to sex. No association was found between the various factors and HBV positivity in males (Table 4). On the other hand, in females, HBV positivity was significantly associated with a history of tattoos and age (Table 5). Multivariate analysis confirmed that both history of tattoos and age were predictive factors for HBV positivity (Table 6).

\section{Discussion}

$\mathrm{HBV}$ is a common public health issue worldwide. Chronic HBV infection is associated with serious complications, such as liver cirrhosis, hepatocellular carcinoma, and hepatic failure. Studies conducted in the Middle East found that the prevalence of HBsAg positivity ranged from less than $3 \%$ in Iraq and some regions in Iran to approximately $7 \%$ in war-torn Yemen and some districts in the Arab peninsula ${ }^{3,4,7}$. Specifically, the results of studies conducted in Iraq varied depending on the recruited samples and the geographic regions. In a previous study in Duhok City, Northern Iraq, the prevalence of HBV was $1.14 \%$ in blood donors ${ }^{8}$, while it was $0.7 \%$ in the middle region of Iraq9. In contrast, in a report from Kerbala, Southern Iraq, HBV had a prevalence of $3.5 \%^{7}$. The vast majority of previous studies in Iraq exclusively recruited male blood donors from one center or city. In this study, the prevalence of HBV was $1.37 \%$, which reflected more realistic results in the general population because both sexes were recruited from three different cities.

\begin{tabular}{lccc}
\hline \multicolumn{3}{l}{ Table 1. Characteristics of the study population } \\
\hline \multicolumn{1}{l}{ Variables } & No. & Percentage \\
\hline Sex & Male & 872 & 25.47 \\
& Female & 2551 & 74.53 \\
Marital status & Married & 3024 & 88.34 \\
& Single & 399 & 11.66 \\
Blood transfusion & Yes & 616 & 17.99 \\
& No & 2807 & 82.01 \\
Surgical operation & Yes & 813 & 23.75 \\
& No & 2610 & 76.25 \\
Dental procedures & Yes & 2228 & 65.09 \\
& No & 1195 & 34.91 \\
Tattoo & Yes & 269 & 7.86 \\
& No & 3154 & 92.14 \\
Injections & Yes & 2017 & 58.92 \\
& No & 1406 & 41.08 \\
Age (Year \pm STD) & & $28.94 \pm 11.17$ & \\
\hline
\end{tabular}

STD: standard deviation

Table 2. Univariate analysis of the hepatitis B virus-associated risk factors in the recruited population

\begin{tabular}{lccccc}
\hline Factors & $\begin{array}{c}\text { HBsAg-Positive } \\
(\boldsymbol{n}=\mathbf{4 7})\end{array}$ & $\begin{array}{c}\text { HBsAg-Negative } \\
(\boldsymbol{n}=\mathbf{3 3 7 6})\end{array}$ & Confidence interval & Odds ratio & $\boldsymbol{P}$-value \\
\hline Sex (Male) & 11 & 861 & $0.4522-1.7604$ & 0.89 & 0.7 \\
Marital status (Married) & 45 & 2979 & $0.7253-12.4205$ & 3 & 0.07 \\
Blood transfusion & 14 & 602 & $1.0398-3.6754$ & 1.95 & 0.048 \\
Surgical operation & 8 & 805 & $0.3053-1.4094$ & 0.66 & 0.25 \\
Dental procedure & 33 & 2195 & $0.6760-2.3792$ & 1.27 & 0.45 \\
Tattoo & 8 & 261 & $1.1323-5.2934$ & 2.44 & 0.039 \\
Injection & 30 & 1987 & $0.6777-2.2454$ & 1.23 & 0.5 \\
Age (Year \pm STD) & $34.42 \pm 11.04$ & $28.87 \pm 11.15$ & $1.0136-1.0579$ & 1.03 & 0.003 \\
\hline
\end{tabular}


HBV can be transmitted via blood and blood product transfusion, exposure to contaminated blood through needles, tattoos, vertically from mothers to newborns (particularly during delivery), and unprotected $\operatorname{sex}^{5}$. It was previously found that the mode of HBV transmission varied in different countries according to age, sex, norms, and societal traditions ${ }^{10}$. It is worth mentioning that a blood screening program for blood-borne diseases started in 2007 in the Kurdistan region. In our study, there was a trend of higher HBV positivity in people with a history of blood transfusion. Careful examination of our data showed that all HBV-positive patients were born before 2007, possibly implying that screening programs in these cities have successfully prevented the transmission of the virus. In agreement with a study conducted in China ${ }^{11}$, we found a significant association between age and HBV positivity. Similarly, a study from Iran showed that HBV positivity was more common in older patients ${ }^{3}$. Again, this could be explained by the fact that the screening program started in 2007, and older patients had a higher chance of exposure. In addition, HBV vaccination was included in the expanded

Table 3. Multivariate analysis of the hepatitis B virusassociated risk factors in the recruited population

\begin{tabular}{lcccc}
\hline Factors & Coef & SE Coef & T value & P-value \\
\hline Age & 0.00056 & 0.0002 & 2.96 & 0.003 \\
Blood transfusion & 0.009 & 0.005 & 1.69 & 0.091 \\
Tattoo & 0.017 & 0.008 & 2.13 & 0.033 \\
\hline
\end{tabular}

Coef: coefficient, SE Coef: standard error of the coefficient. vaccination program in 2000. Therefore, people born before the $\mathrm{HBV}$ vaccination program had a higher chance of infection.

Tattoos are also a risk factor for HBV infection. In a meta-analysis investigating the relationship between HBV and tattooing, tattooing was found to be a risk factor for acquiring the infection ${ }^{12,13}$. Similarly, we found a significant association between HBV positivity and tattooing. This is a challenge for controlling HBV in the Kurdistan region as tattooing is performed in illegitimate shops or as traditions by untrained personnel.

In a study conducted in Turkey, sexual contact with HBV-positive patients was the main risk factor associated with HBV infection ${ }^{14}$. Illegitimate sexual contact could not be included in the study because the ethics committee prohibited the inclusion of questions probing this in the questionnaire since it is a sensitive issue in our conservative society, particularly among females. We believe that even if questions related to this topic were included, the patients would not have provided honest answers.

Previously, the vast majority of studies from Iraq recruited blood donors ${ }^{7,9,15,16}$. Careful examination showed that all donors were male. Therefore, we believe that the majority of studies in this country had not investigated the HBV-related risk factors in females. Therefore, we stratified our data according to sex. We found no association between HBVrelated risk factors and HBV positivity in males. On the other hand, both univariate and multivariate analyses showed that tattoos and age were associated with HBV positivity in females. These results were difficult to explain, necessitating further studies to explore this.

\begin{tabular}{|c|c|c|c|c|c|}
\hline Factors & $\begin{array}{l}\text { HBsAg-Positive } \\
(\mathbf{n}=11)\end{array}$ & $\begin{array}{l}\text { HBsAg-Negative } \\
\quad(n=861)\end{array}$ & Confidence interval & $\begin{array}{l}\text { Odds } \\
\text { ratio }\end{array}$ & $P$-value \\
\hline Marital status & 11 & 598 & $0-0$ & 23 & 0.9 \\
\hline Blood transfusion & 3 & 98 & $0.7619-11.1889$ & 2.9 & 0.153 \\
\hline Surgical operation & 2 & 361 & $0.0661-1.4330$ & 0.3 & 0.094 \\
\hline Dental procedure & 8 & 539 & $0.4196-6.0479$ & 1.59 & 0.5 \\
\hline Tattoo & 1 & 95 & $0.1021-6.3685$ & 0.8 & 0.8 \\
\hline Age (Year \pm STD) & $45.16 \pm 14.4$ & $31.5 \pm 19$ & $0.9950-1.0752$ & 1.03 & 0.09 \\
\hline Injection & 5 & 334 & $0.3981-4.3425$ & 1.31 & 0.65 \\
\hline
\end{tabular}

\begin{tabular}{lccccc}
\hline Table 5. Univariate analysis of the hepatitis B virus-associated risk factors in females & & \\
\hline Factors & $\begin{array}{c}\text { HBsAg-Positive } \\
(\mathbf{n}=\mathbf{3 6})\end{array}$ & $\begin{array}{c}\text { HBsAg-Negative } \\
(\mathbf{n = 2 5 1 5 )}\end{array}$ & Confidence interval & 0dds ratio & P-value \\
\hline Marital status & 34 & 2378 & $0.2277-4.0297$ & 0.95 & 0.9 \\
Blood transfusion & 11 & 504 & $0.8581-3.5918$ & 1.76 & 0.13 \\
Surgical operation & 6 & 444 & $0.3868-2.2598$ & 0.94 & 0.9 \\
Dental procedure & 25 & 1656 & $0.5773-2.4073$ & 1.18 & 0.6 \\
Tattoo & 7 & 166 & $1.4742-7.9139$ & 3.4 & 0.011 \\
Age (Year \pm STD) & $32.63 \pm 9.45$ & $28.41 \pm 8.88$ & $1.0135-1.0754$ & 1.04 & 0.008 \\
Injection & 25 & 1653 & $0.5804-2.4201$ & 1.19 & 0.6 \\
\hline
\end{tabular}




\begin{tabular}{|c|c|c|c|c|}
\hline Factors & Coef & SE Coef & T value & $P$-value \\
\hline Age & 0.00067 & 0.0002 & 2.54 & 0.011 \\
\hline Blood transfusion & 0.01 & 0.005 & 1.65 & 0.1 \\
\hline Tattoo & 0.01 & 0.009 & 2.79 & 0.005 \\
\hline
\end{tabular}

Coef: coefficient, SE Coef: standard error of the coefficient.

The strength of our study was the relatively large and diverse sample, as the majority of previous studies recruited specific populations, such as blood donors or patients on renal dialysis ${ }^{8,17}$. As for its limitation, we only recruited patients who were visiting the said hospitals, rather than random patients in the community. Therefore, a populationbased study is needed to investigate the prevalence and risk factors of $\mathrm{HBV}$ infection. This project is of exceptional importance for public health providers in the region because, to the best of our knowledge, this was the first study to recruit samples from more than one city with a relatively large sample size.

\section{Conclusion}

In conclusion, the prevalence of HBV infection was $1.37 \%$. Our project showed that a history of tattooing and older age were predictive of HBV infection. Tattooing centers in the region should be legalized, trained, and monitored to prevent the further spread of the infection. Screening for HBV infection should focus on people born before 2000 .

\section{Conflict of Interest}

None.

\section{References}

1. El-Serag HB. Epidemiology of Viral Hepatitis and Hepatocellular Carcinoma. Gastroenterology. 2012;142(6):1264-73.e1.

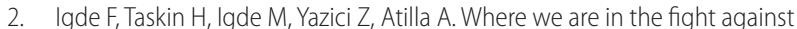
Hepatitis B Infection; Trends in Hepatitis B virus seroprevalence in Black Sea Region of Turkey. Nigerian Journal of Clinical Practice. 2018;21(1):87-92.

3. Alavian SM, Tabatabaei SV, Ghadimi T, Beedrapour F, Kafi-abad SA, Gharehbaghian A, et al. Seroprevalence of Hepatitis B Virus Infection and Its Risk Factors in the West of Iran: A Population-based Study. International Journal of Preventive Medicine. 2012;3(11):770-5.

4. Mohammed Abdullah S. Prevalence of Hepatitis B and C in Donated Blood from the Jazan Region of Saudi Arabia. The Malaysian Journal of Medical Sciences : MJMS. 2013;20(2):41-6.

5. Shepard CW, Simard EP, Finelli L, Fiore AE, Bell BP. Hepatitis B Virus Infection: Epidemiology and Vaccination. Epidemiologic Reviews. 2006;28(1):112-25.

6. Mohammed Saleem ZS, Naqid IA, Hussein NR, Mohammad SJ, Noaman JS, Haji RS, et al. The Prevalence of Hepatitis B and C Virus in Patients With End-Stage Kidney Disease on Regular Hemodialysis in Duhok, Iraq: A Brief Report. Avicenna J Clin Microbiol Infect. 2020;7(1):31-3

7. Assafi MS, Ibrahim NM, Hussein NR, Taha AA, Balatay AA. Urinary bacterial profile and antibiotic susceptibility pattern among patients with urinary tract infection in duhok city, kurdistan region, Iraq. International Journal of Pure and Applied Sciences and Technology. 2015;30(2):54.

8. Hussein NR. Risk factors of hepatitis B virus infection among blood donors in Duhok city, Kurdistan Region, Iraq. Caspian journal of internal medicine. 2018;9(1):22.

9. Al - Juboury A, M. Salih H, AL- ASSADI M, Ali AM. Seroprevalence of Hepatitis B and C among Blood Donors in Babylon Governorate-Iraq. Medical Journal of Babylon 2010;7(1-2)
10. Hussein NR, Daniel S. A Study of Hepatitis B Virus Associated Risk Factors in Patients Attending Hepatitis Unit in Duhok City, Iraq. Archives of Clinical Infectious Diseases. 2017:12(3).

11. Li X, Zheng Y, Liau A, Cai B, Ye D, Huang F, et al. Hepatitis B virus infections and risk factors among the general population in Anhui Province, China: an epidemiological study. BMC Public Health. 2012;12(1):1-7.

12. Jafari S, Buxton JA, Afshar K, Copes R, Baharlou S. Tattooing and risk of hepatitis B: a systematic review and meta-analysis. Can J Public Health. 2012;103(3):207-12

13. Galles NC, Liu PY, Updike RL, Fullman N, Nguyen J, Rolfe S, et al. Measuring routine childhood vaccination coverage in 204 countries and territories, 1980-2019: a systematic analysis for the Global Burden of Disease Study 2020, Release 1. The Lancet. 2021;398(10299):503-21.

14. Ozer A, Yakupogullari Y, Beytur A, Beytur L, Koroglu M, Salman F, et al. Risk factors of hepatitis $B$ virus infection in Turkey: A population-based, casecontrol study. Hepat Mon. 2011:11(4):263-8.

15. Hussein NR, Haj SM, Almizori LA, Taha AA. The prevalence of hepatitis B and C viruses among blood donors attending blood bank in Duhok, Kurdistan region, Iraq. Int J Infect. 2017;4(1):e39008.

16. Jamal SA, Naqid IA, Hussein NR, Abdulqader SR, Nimet AA, Abdulkhdair $H A$, et al. The Prevalence of Hepatitis B and C Virus Infections in the Couples Attending a Premarital Screening Program in Zakho City, Kurdistan Region of Iraq. Zahedan J Res Med Sci. 2020;23(2):e99405.

17. Hussein NR, Saleem ZS, Ibrahim NM, Assafi MS, Daniel S.

The Prevalence of HBV Infection in Renal Transplant Recipients and the Impact of Infection on Graft Survival. Acta Medica Iranica. 2019;57(6):381-4 